\title{
A New and Efficient Nonlinear Solver for Load Flow Problems
}

\author{
Amjad Hussain Khoso \\ Department of Electrical Engineering \\ Mehran University of Engineering and \\ Technology \\ Jamshoro, Pakistan \\ khosoamjadhussain@gmail.com
}

\author{
Muhammad Mujtaba Shaikh \\ Department of Basic Sciences and Related \\ Studies, Mehran University of Engineering \\ and Technology \\ Jamshoro, Pakistan \\ mujtaba.shaikh@faculty.muet.edu.pk
}

\author{
Ashfaque Ahmed Hashmani \\ Department of Electrical Engineering \\ Mehran University of Engineering and \\ Technology \\ Jamshoro, Pakistan \\ ashfaquehashmani1@gmail.com
}

\begin{abstract}
Load Flow (LF) analysis is a fundamental and significant issue in electric power systems. Because of the nonlinearity of the power mismatch equations, the accuracy of the nonlinear solvers is important. In this study, a novel and efficient nonlinear solver is proposed with active applications to LF problems. The formulation of the Proposed Method (PM) and its workflow and mathematical modeling for its application in LF problems have been discussed. The performance of the PM has been validated on the IEEE 14-bus and 30-bus test systems against several existing methods. The simulation results show that the PM exhibits higher order accuracy, faster convergence characteristics, smaller number of iterations, and lesser computation times in comparison with the other benchmark methods.
\end{abstract}

Keywords-electric power system; load flow; Gauss-Seidel; NewtonRaphson; fast decouple; power perturb; efficient method

\section{INTRODUCTION}

Most mathematical models arising in science and engineering are complex and nonlinear in nature. Nonlinear models relate the unknown quantity implicitly within an equation or a system of equations. Such models cannot be solved using traditional analytical methods, and demand the use of efficient and stable numerical methods to yield the approximate solutions to the desired accuracy [1, 2]. Such models arise in different areas of science and engineering, for example the Colebrook's equation is a nonlinear model to find pipe flow systems friction factor implicitly when pipe relative roughness and flow Reynolds number are known [3, 4], chemical combustions problem, NASA's wind satellite problem [1], and the power flow analysis model of a system comprising of buses [5]. LF study is the constant flow analysis of a power system. LF study is important for existing power systems and consequent optimum performance, and is crucial in planning to expand power systems. LF studies serve as a basis in different aspects associated with power systems, for example power flow optimization, continuation and online transmission [5-7]. New methods with robust solutions and fast convergence are still searched and these fast methods for the LF problems are needed in the field of power systems, particularly for optimal power factor extraction and improvement, maximum power point tracking in solar photovoltaic systems [8], power electronics with stability and control [7, 9], steady-state and transient stability of power systems [10-13]. One of the old methods for solving the LF problem was the Gauss-Siedel (GS) method [5, 14-15]. The main drawbacks of the GS method are that it requires a large number of iterations and that it converges slowly. Due to these drawbacks, another most commonly used method is the Newton-Raphson (NR) method, which was proposed for LF studies to overcome the convergence problems of the GS [1416]. In industrial applications, NR method is used widely and it is known as the standard LF method. But, NR method's ability of fast convergence starts to deteriorate when the size of the power system increases. For improving the convergence of the NR method, new methods such as Fast Decoupled (FD) and Power Perturb (PP) [17-18] methods became popular.

In this paper, a new and efficient nonlinear solver for scalar nonlinear equations and vector systems is proposed. The application of the PM has been done on the LF problem. The performance of the PM is compared with different widely used benchmark LF methods on IEEE-14 and IEEE-30 bus standard test systems [19]. Through numerical simulations and results, the ascendancy of the PM over GS, NR, FD, and PP methods has been established from the view-points of higher order accuracy, lower power mismatch errors, lesser number of iterations, and computational time.

\section{LOAD FLOW PROBLEM FORMULATION}

An electric network model of the power system has the objective of efficiently solving voltage and power issues of the network at various buses. The LF study gives information about the magnitude and the angle of the voltage, active power in form of real power, passive component of power, i.e. reactive power and power losses for each bus of the power scheme. The bus-wise equations of power (real and reactive) mismatch can be generally described as:

$$
\begin{gathered}
\Delta P i=P s c h-\sum_{j=1}^{n}|V i||V j||Y i j| \cos (\delta i-\delta j-\theta i j) \\
\Delta Q i=Q s c h-\sum_{j=1}^{n}|V i||V j||Y i j| \sin (\delta i-\delta j-\theta i j)
\end{gathered}
$$

where $\Delta P i=$ mismatch in active power at $i$ bus, $\Delta Q i=$ mismatch in reactive power at $i$ bus, $P s c h=$ injected active power at $i$ bus, $Q s c h=$ injected reactive power at $i$ bus, $V i \angle \delta i=$ 
complex voltage at $i$ bus, $Y i j \angle \theta i j=i j^{\text {th }}$ entry of the admittance matrix, and $n=$ number of power system buses. Consider another equation:

$$
x=\{\delta P V \cup \delta P Q \cup V P Q\}
$$

where $\delta P V$ and $\delta P Q$ represent the vectors of voltage polar angle at $\mathrm{PV}$ and $\mathrm{PQ}$ buses, whereas $V P Q$ is the magnitude of voltage at PQ buses.

Equation (3) defines the unknown array containing the variables of the LF study to be determined. The size of the unknown vector is given by (4):

$$
n x=n P V+2 n P Q
$$

where, $n P V$ and $n P Q$ are the PV and PQ buses numbers, respectively. The iterative algorithm for determining the unknowns is stopped as soon as the pre-specified error tolerance has been achieved, which can be defined in terms of maximum absolute value of mismatch in the powers from (1) and (2), as:

$$
\max \{|\Delta P i| \cup|\Delta Q i|\} \leq \varepsilon \forall i
$$

Equation (5) defines a stopping condition of the LF problem in achieving at most $\varepsilon$ error. In the general form of the nonlinear system, (1) and (2) are usually described as:

$$
g(x)=0
$$

\section{SOME EXISTING METHODS FOR LF PROBLEMS}

\section{A. Gauss-Seidel (GS) Method}

The GS method is well-known as the method of successive approximations. The nonlinear system of equations to be solved can be defined by rearranging (6) as:

$$
x=g(x)
$$

Let $x^{(k)}$ be the starting approximation at $k^{\text {th }}$ iteration for the unknown $x$, then the numerical scheme is

$$
x^{k+1}=g\left(x^{(k)}\right)
$$

When the absolute mismatch of any two consecutive iterates is within the desired accuracy bound $\epsilon$, then the desired solution is obtained [12].

\section{B. Newton-Raphson (NR) Method}

The NR method is used to solve nonlinear scalar equations and systems. Given a particular bus system, its LF is often analyzed by the NR method, which is easy to execute and has simple calculations. To consider the solution of a non-linear algebraic equation, with $x$ as a variable, and if $x^{0}$ is the starting guess for the solution, then the Taylor's series development of $f(x)$ in the vicinity of initial guess can be truncated to the first order derivative term to yield the NR iterative scheme for each iteration $(k)$ to get the new estimated $x$ as:

$$
\begin{gathered}
x^{k+1}=x^{k}-\frac{f\left(x^{k}\right)}{f^{\prime}\left(x^{k}\right)} \\
k=0,1,2, \ldots
\end{gathered}
$$

To solve the nonlinear system (6), we may write (9) as:

$$
x^{(k+1)}=x^{(k)}-J_{x}\left(x^{(k)}\right)^{-1} g\left(x^{(k)}\right)
$$

where $J_{x}$ is an invertible matrix of first order partial derivatives, usually known as the Jacobian matrix. The process defined in (10) continues until the desired accuracy level has been achieved.

\section{PROPOSED METHOD}

Motivated from some recent studies of new time-efficient and convergent nonlinear solvers of different engineering problems $[1,3,4,17]$, a two-step method is proposed which is cubically convergent to solve scalar nonlinear equations, nonlinear systems, and the conventional LF problem arising in electrical power systems.

\section{A. Algorithm of the PM}

The iterative procedure in the PM involves two steps per iteration to solve (6). The PM uses only one additional midpoint of the Jacobian matrix to calculate each iteration when more than one is used in [17]. The main equations of the $\mathrm{PM}$ in scalar form are (11) and (12).

$$
\begin{gathered}
y^{(k)}=x^{(k)}-\frac{f\left(x^{(k)}\right)}{f^{\prime}\left(x^{(k)}\right)} \\
x^{(k+1)}=x^{(k)}-\frac{f\left(x^{(k)}\right)}{f^{\prime}\left(\frac{1}{2}\left[x^{(k)}+y^{(k)}\right]\right)} \\
\text { where } k=0,1, \ldots
\end{gathered}
$$

\section{B. PM Formulation for the LF Problem}

Equations (11) and (12) for (6) become:

$$
y^{(k)}=x^{(k)}+\Delta y^{(k)}
$$

where

$$
\Delta y^{(k)}=-J_{x}\left(x^{(k)}\right)^{-1} g\left(x^{(k)}\right)
$$

and

$$
x^{(k+1)}=x^{(k)}+\Delta x^{(k)}
$$

where

$$
\Delta x^{(k)}=-J_{x}\left(\frac{1}{2}\left[x^{(k)}+y^{(k)}\right]\right)^{-1} g\left(x^{k}\right)
$$

Finally, for nonlinear systems, and hence for the LF problem (1)-(5), the PM can be extended as:

$$
\begin{gathered}
y^{(k)}=x^{(k)}-J_{x}\left(x^{(k)}\right)^{-1} g\left(x^{(k)}\right) \\
x^{(k+1)}=x^{(k)}-J_{x}\left(\frac{1}{2}\left[x^{(k)}+y^{(k)}\right]\right)^{-1} g\left(x^{k}\right)
\end{gathered}
$$

The complete process of the PM when applied to solve LF problems is described by the flow chart of Figure 1. Having obtained the bus and line data of a power system, the PM approaches the solution. The process terminates as soon as the desired accuracy level has been achieved.

\section{RESULTS AND DISCUSSION}

The PM is validated on two well-known bus systems: IEEE 14- and 30-bus test systems' data [18, 19]. Both test systems are parts of the US power system [19]. The 14-bus system 
comprises of 5 positions described by generators and condensers. On the other hand, the 30 -bus system contains a combination of synchronous condensers and generators [18]. The numerical simulations have been performed in MATLAB with standard double precision arithmetic and the error tolerance was set at most $1 \mathrm{E}-08$. The PM and its performance have been compared with other conventional methods: GS, NR, FD and PP.

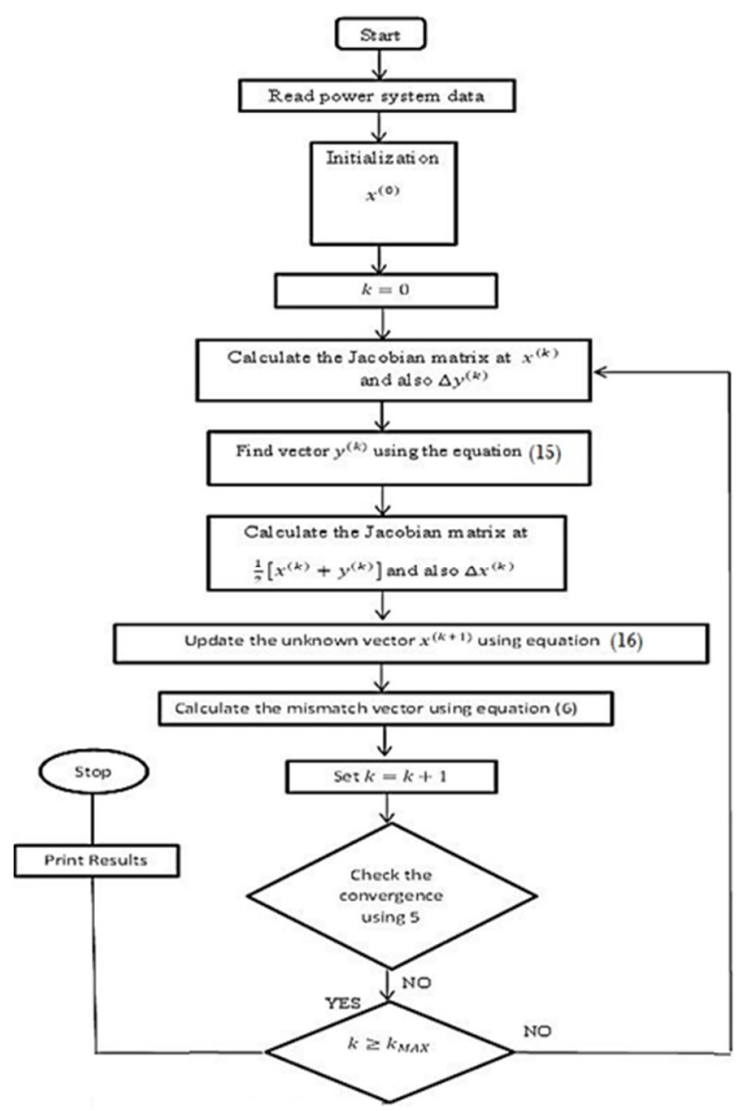

Fig. 1. Flow chart of the PM

The results of the PM for the IEEE 14-bus system are shown in Table I, with details about bus-wise voltage profiles with magnitudes (p.u) and angles (p.u) respectively, along with simulated real and reactive powers across generation and load, in MW and MVar. The comparison of the voltage profiles, in form of magnitude and angles, obtained by the PM and other methods are displayed in Figures 2 and 3 on IEEE 14-bus data, respectively. The PM provides optimal results similar to the other methods' with better precision and accuracy. The voltage magnitudes have been almost higher than those from all other methods. Of all methods, a dissimilar behavior has been observed for PP on IEEE 14-bus data for magnitude and angle of voltages across all buses. For solving the nonlinear voltage equations of IEEE 14-bus power system for the preset error tolerance the PM takes only 6 iterations, whereas NR, PP, FD and GS, respectively require, $11,59,74$, and 182 iterations, as shown in Figure 4. Figure 5 shows the computational time (in seconds) of all methods for the same system. Maximum errors are shown in Figure 6. The NR and PM methods take almost the same execution time on several runs of the program, whereas the highest time was found for the FD method.

TABLE I. PM SIMULATION RESULTS FOR THE IEEE 14-BUS SYSTEM

\begin{tabular}{|c|c|c|c|c|c|c|}
\hline \multirow{3}{*}{ Bus } & \multicolumn{2}{|c|}{ Bus Voltage } & \multicolumn{3}{c|}{ Power (real P and reactive Q) } \\
\cline { 2 - 7 } & $\begin{array}{c}\text { Magnitude } \\
\text { (p.u.) }\end{array}$ & \multirow{2}{*}{$\begin{array}{c}\text { Angle } \\
\text { (p.u.) }\end{array}$} & \multicolumn{2}{c|}{ Generation } & \multicolumn{2}{c|}{ Load } \\
\cline { 4 - 7 } & 1.060 & 0.000 & 159.22 & 38.174 & 30.380 & 17.780 \\
\hline 1 & 1.045 & -1.513 & 232.00 & 0.120 & 0.000 & 0.000 \\
\hline 2 & 1.010 & -13.641 & 0.000 & 63.353 & 131.880 & 26.600 \\
\hline 3 & 0.992 & -10.429 & 0.000 & 0.000 & 66.920 & 10.000 \\
\hline 4 & 0.999 & -8.744 & 0.000 & 0.000 & 10.640 & 2.240 \\
\hline 5 & 1.070 & -17.435 & 0.000 & 60.714 & 15.680 & 10.500 \\
\hline 6 & 1.032 & -15.028 & 0.000 & 0.000 & 0.000 & 0.000 \\
\hline 7 & 1.090 & -15.028 & 0.000 & 35.849 & 0.000 & 0.000 \\
\hline 8 & 1.008 & -17.461 & 0.000 & 0.000 & 41.300 & 23.240 \\
\hline 9 & 1.006 & -17.871 & 0.000 & 0.000 & 12.600 & 8.120 \\
\hline 10 & 1.028 & -17.821 & 0.000 & 0.000 & 4.900 & 2.520 \\
\hline 11 & 1.036 & -18.623 & 0.000 & 0.000 & 8.540 & 2.240 \\
\hline 12 & 1.036 & -18.643 & 0.000 & 0.000 & 18.900 & 8.120 \\
\hline 13 & 1.027 & -19.494 & 0.000 & 0.000 & 20.860 & 7.000 \\
\hline 14 & 0.990 & -190 \\
\hline
\end{tabular}

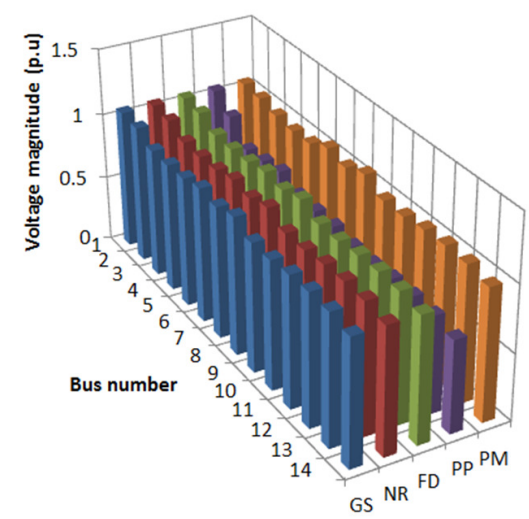

Fig. 2. Comparison of voltage magnitude for IEEE-14 data

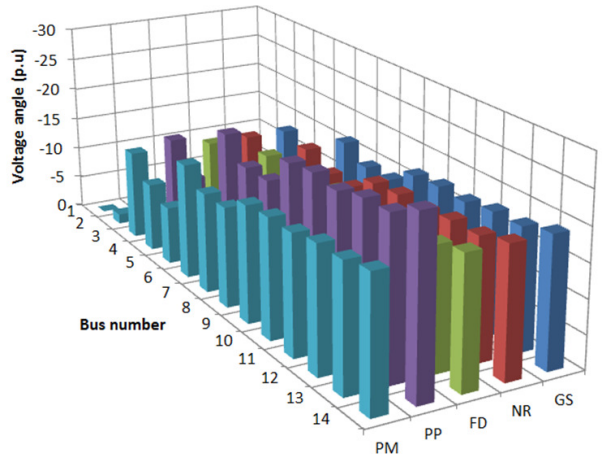

Fig. 3. Comparison of voltage angle for IEEE-14 data

The simulation results for the IEEE 30-bus system by the PM in form of bus-wise voltage magnitudes and voltage angles profiles along with simulated real and reactive powers across generation and load are shown in Table II. The comparison of the voltage magnitude and angles for all methods on the IEEE 30-bus data are shown in Figures 7 and 8, respectively. 


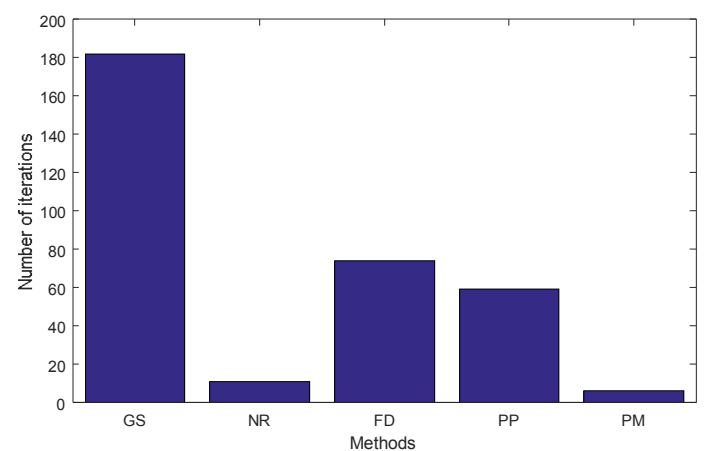

Fig. 4. Iterations taken by all methods for the IEEE 14-bus system

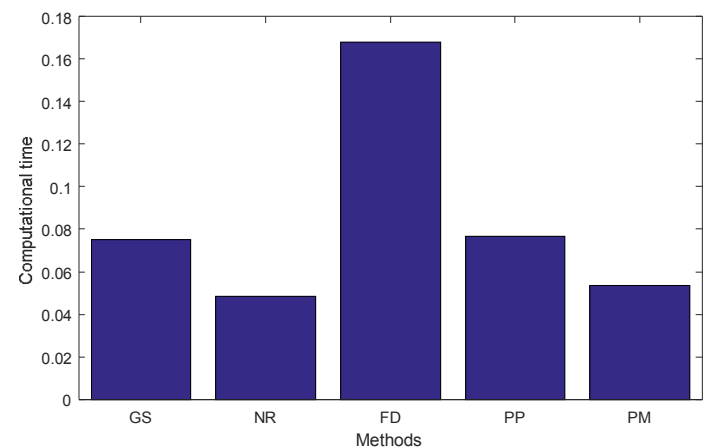

Fig. 5. Computational time (s) by all methods for the IEEE 14-bus system

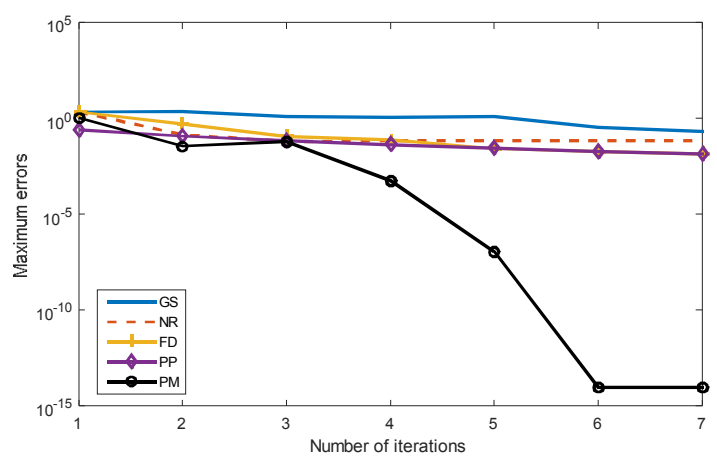

Fig. 6. Maximum errors for all methods for the IEEE 14-bus system

The PM provides optimal results similar to other methods in this case too with better precision and accuracy. The voltage magnitudes have been almost higher than those of the other methods. Again the PP method shows different results for the IEEE 30-bus system in both magnitude and angle voltages across all buses. For solving the nonlinear IEEE 30-bus power system data for the desired error bound, the PM takes only 4 iterations, whereas NR, PP, FD and GS, respectively require, 5, 24, 71, and 93 iterations, as shown in Figure 9. Figure 10 shows the computational time (in seconds) for all methods for the same system, whereas maximum errors are shown in Figure 11. The NR and PM methods take almost the same execution time, with PM taking ascendancy minimum most of the program runs, whereas the highest time was found for the FD method.
TABLE II. PM SIMULATION RESULTS FOR THE IEEE 30-BUS SYSTEM

\begin{tabular}{|c|c|c|c|c|c|c|}
\hline \multirow{2}{*}{ Bus } & \multicolumn{2}{|c|}{ Bus Voltage } & \multicolumn{2}{|c|}{ Power (real P and reactive Q) } \\
\cline { 2 - 7 } & $\begin{array}{c}\text { Magnitude } \\
\text { (p.u) }\end{array}$ & $\begin{array}{c}\text { Angle } \\
\text { (p.u) }\end{array}$ & \multicolumn{2}{|c|}{ Generation } & \multicolumn{2}{c|}{ Load } \\
\hline 1 & 1.060 & 0.000 & 260.99 & -17.021 & 0.000 & 0.000 \\
\hline 2 & 1.043 & -5.497 & 40.000 & 48.822 & 21.700 & 12.700 \\
\hline 3 & 1.022 & -8.004 & 0.000 & 0.000 & 2.400 & 1.200 \\
\hline 4 & 1.013 & -9.661 & 0.000 & 0.000 & 7.600 & 1.600 \\
\hline 5 & 1.010 & -14.381 & 0.000 & 35.975 & 94.200 & 19.000 \\
\hline 6 & 1.012 & -11.398 & 0.000 & 0.000 & 0.000 & 0.000 \\
\hline 7 & 1.003 & -13.150 & 0.000 & 0.000 & 22.800 & 10.900 \\
\hline 8 & 1.010 & -12.115 & 0.000 & 30.826 & 30.000 & 30.000 \\
\hline 9 & 1.051 & -14.434 & 0.000 & 0.000 & 0.000 & 0.000 \\
\hline 10 & 1.044 & -16.024 & 0.000 & 0.000 & 5.800 & 2.000 \\
\hline 11 & 1.082 & -14.434 & 0.000 & 16.119 & 0.000 & 0.000 \\
\hline 12 & 1.057 & -15.302 & 0.000 & 0.000 & 11.200 & 7.500 \\
\hline 13 & 1.071 & -15.302 & 0.000 & 10.424 & 0.000 & 0.000 \\
\hline 14 & 1.042 & -16.191 & 0.000 & 0.000 & 6.200 & 1.600 \\
\hline 15 & 1.038 & -16.278 & 0.000 & 0.000 & 8.200 & 2.500 \\
\hline 16 & 1.045 & -15.880 & 0.000 & 0.000 & 3.500 & 1.800 \\
\hline 17 & 1.039 & -16.188 & 0.000 & 0.000 & 9.000 & 5.800 \\
\hline 18 & 1.028 & -16.884 & 0.000 & 0.000 & 3.200 & 0.900 \\
\hline 19 & 1.025 & -17.052 & 0.000 & 0.000 & 9.500 & 3.400 \\
\hline 20 & 1.029 & -16.852 & 0.000 & 0.000 & 2.200 & 0.700 \\
\hline 21 & 1.032 & -16.468 & 0.000 & 0.000 & 17.500 & 11.200 \\
\hline 22 & 1.033 & -16.455 & 0.000 & 0.000 & 0.000 & 0.000 \\
\hline 23 & 1.027 & -16.662 & 0.000 & 0.000 & 3.200 & 1.600 \\
\hline 24 & 1.022 & -16.830 & 0.000 & 0.000 & 8.700 & 6.700 \\
\hline 25 & 1.019 & -16.424 & 0.000 & 0.000 & 0.000 & 0.000 \\
\hline 26 & 1.001 & -16.842 & 0.000 & 0.000 & 3.500 & 2.300 \\
\hline 27 & 1.026 & -15.912 & 0.000 & 0.000 & 0.000 & 0.000 \\
\hline 28 & 1.011 & -12.057 & 0.000 & 0.000 & 0.000 & 0.000 \\
\hline 29 & 1.006 & -17.136 & 0.000 & 0.000 & 2.400 & 0.900 \\
\hline 30 & 0.995 & -18.015 & 0.000 & 0.000 & 10.600 & 1.900 \\
\hline & & & & & & \\
\hline & & & & & \\
\hline & & & & & & \\
\hline
\end{tabular}

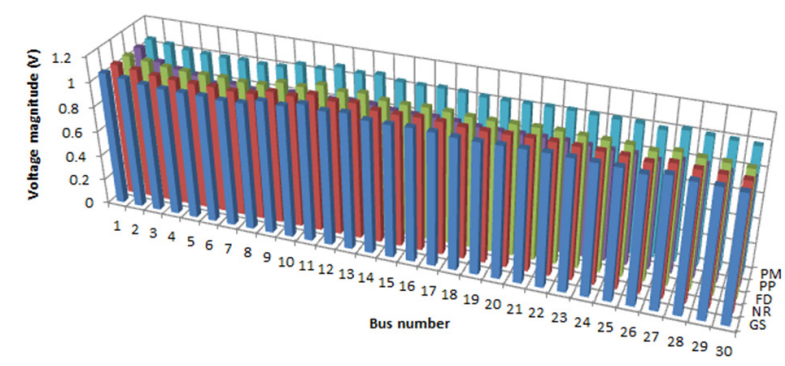

Fig. 7. Comparison of voltage magnitude methods for IEEE-30 data

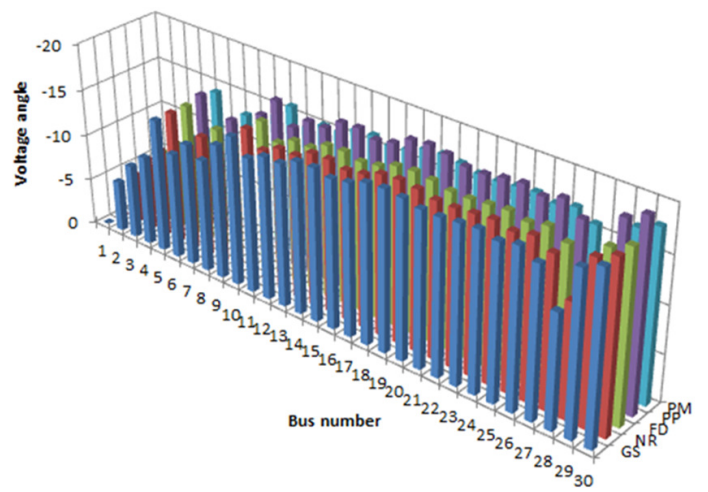

Fig. 8. Comparison of voltage angle by all methods for IEEE-30 data

From the exhaustive simulations and consequent comparison of results, an encouraging performance of the new 
and efficient PM has been established for LF problems with successful validation on IEEE 14 and IEEE 30 test bus systems against conventional methods. The PM exhibits higher order accuracy and better convergence characteristics than known benchmark methods GS, NR, FD and PP. The PM converges quickly, takes lesser computational time, and has a lower iteration count in comparison with the other methods.

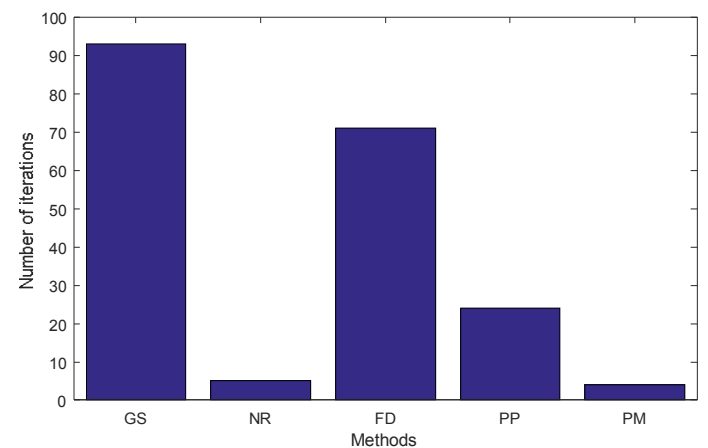

Fig. 9. Iteration number comparison for the IEEE 30-bus system

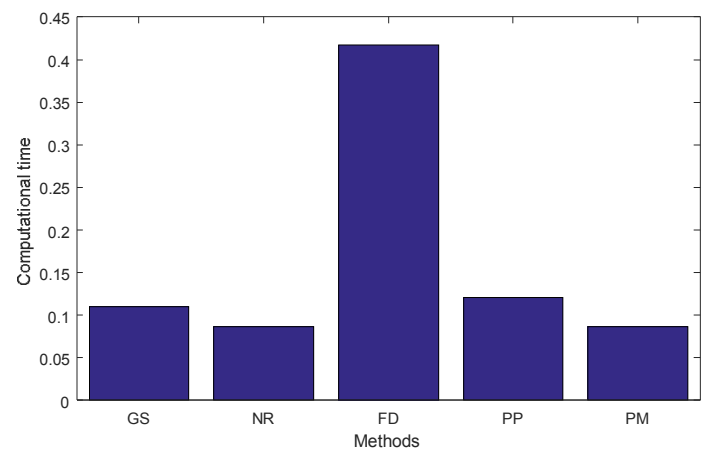

Fig. 10. Computational time comparison for the IEEE 30-bus system

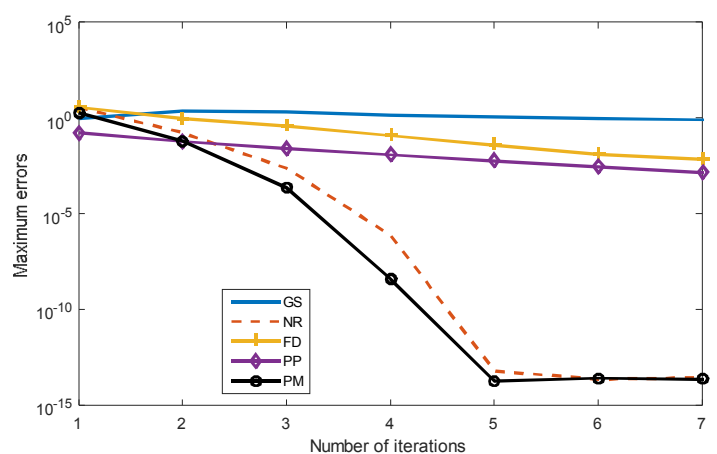

Fig. 11. Maximum errors comparison for the IEEE 30-bus system

\section{CONCLUSION}

In this paper, a new iterative nonlinear solver, referred to as PM, was proposed for LF problems. The mathematical model of PM for solving the LF problems was presented. The structural approach of the PM to efficiently solve nonlinear equations of LF problems was systematically presented and worked out. The performance of the PM against other benchmark LF solvers was tested on IEEE 14- and 30-bus systems' test data, through numerical simulations on MATLAB. The main advantages of our PM are its better convergence properties, less computational time, and smaller iteration number when compared with the standard LF methods.

\section{ACKNOWLEDGEMENT}

The authors would like express their gratitude to the Mehran University of Engineering and Technology authorities for their support.

\section{REFERENCES}

[1] H. A. Abro, M. M. Shaikh, "A new time-efficient and convergent nonlinear solver", Applied Mathematics and Computation, Vol. 355, pp. 516-536, 2019

[2] F. L. Alvarado, "Bifurcations in nonlinear systems-computational issues", IEEE International Symposium on Circuits and Systems, pp. $922-925,1990$

[3] M. M. Shaikh, S. U. R. Massan, A. I. Wagan, "A new explicit approximation to Colebrook's friction factor in rough pipes under highly turbulent cases", International Journal of Heat and Mass Transfer, Vol. 88, No. 3, pp. 538-543, 2015

[4] M. M. Shaikh, S. U. R. Massan, A. I. Wagan, “A sixteen decimal places' accurate Darcy friction factor database using non-linear Colebrook's equation with a million nodes: a way forward to the soft computing techniques", Data in Brief, Vol. 27, Article ID 104733, 2019

[5] B. Stott, "Review of load-flow calculation methods", Proceedings of the IEEE, Vol. 62, pp. 916-929, 1974

[6] V. Ajjarapu, Computational techniques for voltage stability assessment and control, Springer, 2010

[7] P. Kundur, Power system stability and control, McGraw-Hill, 1993

[8] V. Bhan, A. A. Hashmani, M. M. Shaikh, "A new computing perturband-observe-type algorithm for MPPT in solar photovoltaic systems and evaluation of its performance against other variants by experimental validation", Scientia Iranica, Vol. 26, pp. 3656-3671, 2019

[9] N. E. Akpeke, C. M. Muriithi, C. Mwaniki, "Contribution of FACTS devices to the transient stability improvement of a power system integrated with a PMSG-based wind turbine", Engineering, Technology \& Applied Science Research, Vol. 9, No. 6, pp. 4893-4900, 2019

[10] Z. A. Shahani, A. A. Hashmani, M. M. Shaikh, "Steady state stability analysis and improvement using eigenvalues and PSS: A case study of a thermal power plant in Jamshoro, Pakistan", Engineering, Technology \& Applied Science Research, Vol. 10, No. 1, pp. 5301-5306, 2020

[11] O. Kahouli, B. Ashammari, K. Sebaa, M. Djebali, H. H. Abdallah, "Type-2 fuzzy logic controller based PSS for large scale power systems stability", Engineering, Technology \& Applied Science Research, Vol. 8, No. 5, pp. 3380-3386, 2018

[12] G. Shahgholian, A. Fattollahi, "Improving power system stability using transfer function: A comparative analysis", Engineering, Technology \& Applied Science Research, Vol. 7, No. 5, pp. 1946-1952, 2017

[13] D. V. Ngo, K. V. Pham, D. D. Le, K. H. Le, K. V. Huynh, “Assessing power system stability following load changes and considering uncertainty", Engineering, Technology \& Applied Science Research, Vol. 8, No. 2, pp. 2758-2763, 2018

[14] S. Mallick, D. V. Rajan, S. S. Thakur, P. Acharjee, S. P. Ghoshal, "Development of a new algorithm for power flow analysis", International Journal of Electrical Power \& Energy Systems, Vol. 33, No. 8, pp. 1479-88, 2011

[15] M. Ebeed, S. Kamel, F. Jurado, "Determination of IPFC operating constraints in power flow analysis", Electrical Power \& Energy Systems, Vol. 81, pp. 299-307, 2016

[16] W. F. Tinney, C. E. Hart, "Power flow solution by Newton's method", IEEE Transactions on Power Apparatus and Systems, Vol. PAS-86, No. 11, pp. 1449-60, 1967 
[17] M. Tostado, S. Kamel, F. Jurado, "Developed Newton-Raphson based predictor-corrector load flow approach with high convergence rate", International Journal of Electrical Power \& Energy Systems, Vol. 105, pp. 785-792, 2019

[18] H.Saadat, Power System Analysis, Pearson, 2011

[19] University of Washington, Power systems test case archive, available at: https://labs.ece.uw.edu/pstca/

\section{AUTHORS PROFILE}

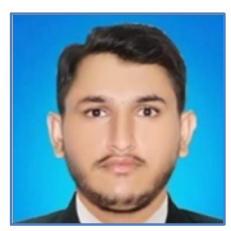

Amjad Hussain Khoso has a BE of Electrical Engineering from Mehran University of Engineering and Technology, Jamshoro, Pakistan. At present, he is pursuing his ME from the same department.

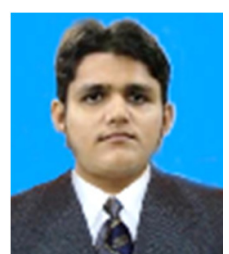

Muhammad Mujtaba Shaikh is working as an Assistant Professor (Mathematics) in the Department of Basic Sciences and Related Studies, Mehran University of Engineering and Technology. He received his $\mathrm{PhD}$ from the Institute of Mathematics and Computer Science, University of Sindh, Jamshoro, Pakistan in 2017. He has been awarded with gold and silver medals in BS (Mathematics) degree in 2009. He is an active researcher having an impact factor of over 20 according to most reputed Journal Citations Reports by Thompson Reuters. He has profound knowledge on computational methods and statistical analysis, and has been involved in teaching and research in these fields. His research fields include optimization, numerical integration, applied and computational mathematics, and mathematical problems in engineering. He is also an active member of Supply Chain and Operations Management (SCOM) research group at Mehran University of Engineering and Technology. Besides, a number of research scholars are working under his supervision in MS, MPhil, ME and PhD programs.

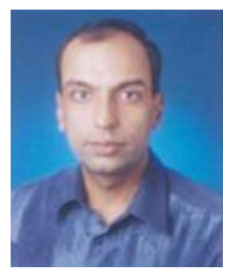

Ashfaque Ahmed Hashmani has a BE from Mehran University of Engineering and Technology, a ME from Singapore, and a $\mathrm{PhD}$ from University of DuisbergEssen. At present, he is a Professor and Chairman at the Department of Electrical Engineering, Mehran University of Engineering and Technology. His fields of interest are: power system stability and control, nonlinear control and applications, robust control of power and energy systems. 